\title{
When is there a multipartite maximum entangled state?
}

\author{
Runyao Duan 2 \\ Centre for Quantum Computation and Intelligent Systems (QCIS), \\ Faculty of Engineering and Information Technology, \\ University of Technology, Sydney (UTS), NSW 2007, Australia \\ and \\ Department of Computer Science and Technology, \\ Tsinghua National Laboratory for Information Science and Technology, \\ Tsinghua University, Beijing 100084, China \\ Yaoyun Shi 3 \\ Department of Electrical Engineering and Computer Science, University of Michigan \\ 2260 Hayward Street, Ann Arbor, MI 48109-2121, USA
}

\begin{abstract}
For a multipartite quantum system of the dimension $d_{1} \otimes d_{2} \otimes \cdots d_{n}, d_{1} \geq d_{2} \geq \cdots \geq d_{n}$, is there an entangled state maximum in the sense that all other states in the system can be obtained from the state through local quantum operations and classical communications (LOCC)? When $d_{1} \geq \Pi_{i=2}^{n} d_{i}$, such state exists. We show that this condition is also necessary. Our proof, somewhat surprisingly, uses results from algebraic complexity theory.
\end{abstract}

Keywords: quantum information theory, quantum communication protocol,tensor rank, maximum entangled state, stochastic entanglement transformation

\footnotetext{
${ }^{1}$ This work was supported in part by the National Science Foundation of the United States under Awards 0347078 and 0622033.

2 Email: Runyao.Duan@uts.edu.au,dry@tsinghua.edu.cn. Supported in part by the National Natural Science Foundation of China (Grant Nos. 60702080 and 60621062), the FANEDD under Grant No. 200755, the Hi-Tech Research and Development Program of China (863 project) (Grant No. 2006AA01Z102), and QCIS of UTS. Part of this work was done while the author was visiting University of Michigan, Ann Arbor.

${ }^{3}$ Email: shiyy@eecs.umich.edu
} 


\section{Background and the statement of the main result}

A quantum system consisting of several subsystems may be in an entangled state, such that measurements on the subsystems may produce outcome statistics fundamentally different from those produced through a classical process. Since its discovery by Einstein, Podolsky, and Rosen [1, quantum entanglement has been found to be central for non-classical properties of quantum systems. In particular, it plays a fundamental role in quantum information processing applications such as unconditional secure key distribution and super fast quantum algorithms. It is therefore of fundamental importance to understand the nature of entanglement. Indeed, the past two decades have witnessed the rapid development of a theory of quantum information, at the heart of which is the theory of quantum entanglement. Horodecki et al. [2] and Gühne and Tóth [3] are recent surveys on the subject.

Our study is motivated by the following objective, which is important for the practical applications of quantum entanglement: how do we establish quantum entanglement between multiple parties separated spatially? One straightforward solution is for one party to prepare the desired state $|\phi\rangle$, and send the others their corresponding portion of the state. The problem of this solution is that moving quantum objects around without corrupting them is difficult and expensive, especially when the parties are remotely separated.

The celebrated quantum teleportation protocol [4] provides an alternative approach: the parties initially share some special but fixed entangled state $\left|\phi_{0}\right\rangle$, which will then be transformed to $|\phi\rangle$ through local quantum operations and classical communications (LOCC). Ideally, $\left|\phi_{0}\right\rangle$ should work for all possible $|\phi\rangle$ desired. The question we address is: for which dimensions of the system is there such an initial state that can generate all other states in the system?

Let $n, d_{1}, d_{2}, \cdots, d_{n}$ be integers with $n \geq 2$, and $d_{1} \geq d_{2} \geq \cdots \geq d_{n} \geq 2$. We denote by $d_{1} \otimes d_{2} \otimes \cdots d_{n}$ the tensor product of $n$ Hilbert spaces, each of the dimension $d_{1}, d_{2}, \ldots$, $d_{n}$, respectively. We refer to the whole system by $H$, and each subsystem by $A, B, C, \ldots$, $Z$, respectively. We use superscripts $A, B, C, \cdots, Z$ on states or operators to indicate the space they are associated with. Let $\left|\phi_{1}\right\rangle$ and $\left|\phi_{2}\right\rangle$ be two states in $d_{1} \otimes \cdots \otimes d_{n}$. We write $\left|\phi_{2}\right\rangle \leq_{\text {LOCC }}\left|\phi_{1}\right\rangle$ if $\left|\phi_{1}\right\rangle$ can be transformed to $\left|\phi_{2}\right\rangle$ through a LOCC protocol. A state $\left|\phi_{0}\right\rangle$ is said to be a maximum entangled state (MES) if $|\phi\rangle \leq_{\text {LOCC }}\left|\phi_{0}\right\rangle$ for all $|\phi\rangle$ in the same space. Thus our problem is, which space $d_{1} \otimes \cdots \otimes d_{n}$ contains a maximum entangled state?

Besides the practical motivation described above, our question is also among the most basic questions in the framework of entanglement manipulations, which is to study properties of entanglement under LOCC transformations. This is a major paradigm for studying entanglement where many central results were obtained. A particular task in this paradigm is to classify entangled states through their conversion relations. Note that classical communication should not increase any reasonable notion quantifying entanglement - indeed, this monotonicity under LOCC transformation is considered the only natural requirement for entanglement measures [5]. Therefore, the relation $\leq_{\text {LOCC }}$ induces a natural partial ordering of quantum states (or more precisely, of the LOCC equivalence classes) by the amount of entanglement. Our problem, which is to ask when a maximum element exists, is thus among the very basic questions regarding the structure of this ordering. We stress that the definition of "maximum" in this paper is restricted to the LOCC ordering. There may be other definitions of maximum entangled states with respect to other orderings. 
When $n=2$, the answer to our question is well known through the use of the teleportation protocol with the generalized EPR state (commonly referred to as the maximum entangled state for bipartite systems)

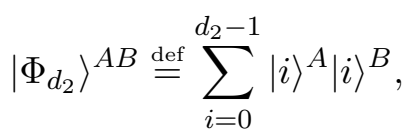

where $\left\{|i\rangle^{A}: i=0 . . d_{2}-1\right\}$ and $\left\{|i\rangle^{B}: i=0 . . d_{2}-1\right\}$ are orthonormal in $A$ and $B$, respectively. The teleportation protocol can be generalized to arbitrary $n$, as long as

$$
d_{1} \geq \Pi_{i=2}^{K} d_{i} .
$$

On the other hand, not all spaces have a maximum entangled state. For example, Dür, Vidal and Cirac showed that there is no MES in the $2 \otimes 2 \otimes 2$ space [6]. The main result of this paper is that a MES exists only if Eqn. (2) holds.

Our result is actually slightly stronger. Following the notation of Bennett et al. 7], if $\left|\phi_{1}\right\rangle$ can be transformed to $\left|\phi_{2}\right\rangle$ with a non-zero probability, we write $\left|\phi_{2}\right\rangle \leq_{\text {SLOCC }}\left|\phi_{1}\right\rangle$, where "SLOCC" stands for Stochastic Local Operations and Classical Communications. Similarly, $\left|\phi_{1}\right\rangle$ is called a stochastic maximum entangled state if $\left|\phi_{2}\right\rangle \leq_{\text {SLOCC }}\left|\phi_{1}\right\rangle$ for all $\left|\phi_{2}\right\rangle$ in the same space. The partial ordering $\leq_{\mathrm{SLOCC}}$ was introduced by Bennett et al. [7] in order to provide a simpler classification of multipartite entanglement (there are infinitely number of LOCC equivalence classes even for 2 qubits), and has been subsequently studied by many authors. Clearly a MES is also a SMES; thus if Eqn. (2) holds then a SMES exists. We now state our main theorem.

Theorem 1.1. If $d_{1}<\prod_{i=2}^{n} d_{i}$, there is no stochastic maximum entangled state in the state space $d_{1} \otimes d_{2} \otimes \cdots \otimes d_{n}$.

Our proof uses the notion of tensor rank from algebraic complexity theory (C.f. Chapter 14 in [8]). The tensor rank of $|\phi\rangle \in \mathcal{H}, S c h(\phi)$, is the minimum number of product vectors that can linearly express $|\phi\rangle$. That is, $S c h(\phi)$ is the minimum integer $k$ such that there exists product vectors $\left|\phi_{i}^{A}\right\rangle \otimes\left|\phi_{i}^{B}\right\rangle \otimes \cdots\left|\phi_{i}^{Z}\right\rangle \in d_{1} \otimes d_{2} \otimes \cdots \otimes d_{n}$ such that

$$
|\phi\rangle \stackrel{\text { def }}{=} \sum_{i=1}^{k}\left|\phi_{i}^{A}\right\rangle \otimes\left|\phi_{i}^{B}\right\rangle \otimes \cdots\left|\phi_{i}^{Z}\right\rangle .
$$

$\operatorname{Sch}(\phi)$ can also be called Schmidt rank or Schmidt number [9], and is precisely the rank of the reduced density matrix $\operatorname{Tr}_{A}(|\phi\rangle\langle\phi|)$ when $n=2$. In general, the tensor rank is the minimum number of multiplications to compute a set of linear forms determined by $|\phi\rangle$. For example, the minimum number of non-scalar multiplications for multiplying two $n$ by $n$ matrices is precisely the tensor rank of the following element in $n^{2} \otimes n^{2} \otimes n^{2}$ :

$$
\sum_{i, j, k=0}^{n-1}|i, j\rangle|i, k\rangle|k, j\rangle,
$$

where each component space has a product orthonormal basis $\{|i, j\rangle: i, j=0 . . n-1\}$. It was observed in [10] that the above state is precisely the tripartite state $\left|\Psi_{n}\right\rangle^{A B C}=\left|\Phi_{n}\right\rangle^{A B} \otimes$ 
$\left|\Phi_{n}\right\rangle^{B C} \otimes\left|\Phi_{n}\right\rangle^{C A}$. This connection enables us and a co-author to show the equivalence between the computational complexity of matrix multiplication and efficiency of a certain entanglement transformation that produces EPR pairs [10].

The tensor rank of a Hilbert space $\mathcal{H}$ is

$$
\operatorname{Sch}(\mathcal{H}) \stackrel{\text { def }}{=} \max \{S \operatorname{sch}(\phi):|\phi\rangle \in \mathcal{H}\} .
$$

Many works have been done to determine the tensor rank of specific tensors and of various spaces. We will use the following results.

Theorem 1.2. Consider $\operatorname{Sch}(\mathcal{H})$ for $\mathcal{H}=d_{1} \otimes d_{2} \otimes d_{3}$. Let $k=d_{2} d_{3}-d_{1}$.

(i) (Theorem 6(ii) of [11]) If $k \geq 1$, then $\operatorname{Sch}(\mathcal{H}) \geq d_{1}+\lfloor\sqrt{2 k+2}\rfloor-2$.

(ii) (Theorem 3 of [12]) If $k \leq \max \left\{d_{2}, d_{3}\right\}$ and $0 \leq k \leq 4$, then $\operatorname{Sch}(\mathcal{H})=d_{2} d_{3}-\left\lceil\frac{k}{2}\right\rceil$.

\section{Proof of the Main Theorem}

We now turn to the proof of the main result. We shall first obtain some structural results about SLOCC and the induced ordering on the states. We say that $\left|\phi_{1}\right\rangle$ and $\left|\phi_{2}\right\rangle$ are SLOCC equivalent if $\left|\phi_{1}\right\rangle \leq_{\text {SLOCC }}\left|\phi_{2}\right\rangle$ and $\left|\phi_{2}\right\rangle \leq_{\text {SLOCC }}\left|\phi_{1}\right\rangle$. Then $\leq_{\text {SLOCC }}$ defines a partial oder on SLOCC equivalence classes. We will often identify a state with its equivalence class. A state $|\phi\rangle$ is said to be SLOCC maximal if for any $|\psi\rangle,|\phi\rangle \leq_{\text {SLOCC }}|\psi\rangle$ implies $|\psi\rangle \leq_{\text {SLOCC }}|\phi\rangle$. For the rest of the paper, we may omit "SLOCC" when referring to equivalence, equivalence classes, maximal state, etc. We know the following fact about SLOCC [6].

Lemma 2.1. Let $|\phi\rangle$ and $|\psi\rangle \in d_{1} \otimes d_{2} \cdots \otimes d_{n}$. Then $|\psi\rangle \leq_{\mathrm{SLOCC}}|\phi\rangle$ if and only if there are linear operators $L_{1}, \cdots, L_{n}$ such that $\left(L_{1} \otimes \cdots \otimes L_{n}\right)|\phi\rangle=|\psi\rangle$. In particular, $|\phi\rangle$ and $|\psi\rangle$ are equivalent under SLOCC if and only if $L_{1}, \cdots, L_{n}$ are invertible.

Since local linear operators cannot increase tensor rank, we have the following fact that relates tensor rank and SLOCC [13].

Proposition 2.2. If $|\psi\rangle \leq_{S L O C C}|\phi\rangle, S \operatorname{ch}(\phi) \geq S \operatorname{ch}(\psi)$.

We say that $|\Phi\rangle \in \mathcal{H}$ is of full local ranks if $\operatorname{rank}\left(\rho_{\Phi}^{k}\right)=d_{k}$ for any $k, 1 \leq k \leq n$, where $\rho_{\Phi}^{k}$ is the reduced density operator of $|\Phi\rangle\langle\Phi|$ obtained by tracing out all subsystems other than the $k$ 'th one. We characterize maximal states below.

Lemma 2.3. A state is maximal if and only if it has full local ranks.

Proof. We prove the result for $n=3$. The other cases are similar. Suppose that $|\Phi\rangle$ is of full local ranks. Let $|\Psi\rangle \in \mathcal{H}$ be such that $|\Phi\rangle \leq_{\text {SLOCC }}|\Psi\rangle$. Then there exists linear operators $L_{1}, L_{2}, L_{3}$ such that $\left(L_{1} \otimes L_{2} \otimes L_{3}\right)|\Psi\rangle=|\Phi\rangle$. As $|\Phi\rangle$ is of full local ranks, we have that $L_{1}, L_{2}, L_{3}$ should be invertible. Thus $|\Psi\rangle$ and $|\Phi\rangle$ are equivalent, implying that $|\Phi\rangle$ is maximal. 
For the other direction, assume for the purpose of getting a contradiction that $|\Phi\rangle$ is maximal but is not of full local ranks. Without loss of generality, assume that $\operatorname{rank}\left(\rho_{\Phi}^{1}\right)=$ $k<d_{1}$. Let $\left\{|i\rangle^{A}: 1 \leq i \leq k\right\}$ be an orthonormal basis for the support of $\rho_{\Phi}^{A}$. Write

$$
|\Phi\rangle=\sum_{i=1}^{k}|i\rangle^{A}\left|\phi_{i}\right\rangle^{B C} .
$$

Let $|\psi\rangle^{A} \in A$ be such that $\langle i \mid \psi\rangle=0$ for all $i, 1 \leq i \leq k$. Construct $\left|\Phi^{\prime}\right\rangle$ as follows:

$$
\left|\Phi^{\prime}\right\rangle=|\Phi\rangle+|\psi\rangle^{A}|\phi\rangle^{B C}
$$

where $|\phi\rangle^{B C}$ is any nonzero vector. Let $P^{A}=\sum_{i=1}^{k}|i\rangle\langle i|$. One can easily verify that $\left|\Phi^{\prime}\right\rangle$ can be transformed into $|\Phi\rangle$ by SLOCC as

$$
\left(P^{A} \otimes I^{B} \otimes I^{C}\right)\left|\Phi^{\prime}\right\rangle=|\Phi\rangle .
$$

However, $\left|\Phi^{\prime}\right\rangle$ is not equivalent to $|\Phi\rangle$ as $\operatorname{rank}\left(\rho_{\Phi^{\prime}}^{A}\right)=k+1>\operatorname{rank}\left(\rho_{\Phi}^{A}\right)$. That contradicts the fact that $|\Phi\rangle$ is maximal.

The partition of the space $d_{1} \otimes \cdots \otimes d_{n}$ into $n$ sub-systems may be further refined by partitioning one, or several, sub-system $d_{i}$ into a product space $d_{i, 1} \otimes d_{i, 2} \otimes \cdots \otimes d_{i, k_{i}}$, where $\Pi_{j=1}^{k_{i}} d_{i, j}=d_{i}$. Note that if a density operator $\rho^{i}$ on the $i^{\prime}$ th sub-system is of full rank, its reduced density operator $\rho^{i, j}, j=1 . . k_{j}$ on the $j^{\prime}$ th sub-system in the refinement is also of full rank. Thus we have the following useful consequence of the above lemma.

Corollary 2.4. A maximal state remains maximal with respect to a refined partition.

The following lemma shows that there are at least two general ways of constructing a maximal state.

Lemma 2.5. There is a maximal state $|\Phi\rangle$ such that $S \operatorname{ch}(\Phi)=S \operatorname{ch}(\mathcal{H})$. There is also a maximal state with tensor rank $d_{1}$.

Proof. By definition, there exists $|\Phi\rangle$ such that $S \operatorname{ch}(\Phi)=S \operatorname{ch}(\mathcal{H})$. So we can write

$$
|\Phi\rangle=\sum_{i=1}^{S c h(\mathcal{H})}\left|\alpha_{i}\right\rangle\left|\beta_{i}\right\rangle\left|\gamma_{i}\right\rangle .
$$

If $|\Phi\rangle$ is of full local rank, then it follows from the previous lemma that $|\Phi\rangle$ is maximal. Otherwise, assume without loss of generality that $\operatorname{rank}\left(\rho_{\Phi}^{A}\right)<d_{1}$. Thus there exists another nonzero vector $\left|a_{1}\right\rangle \in \mathcal{H}_{A}$ such that $\left\langle a_{1} \mid \alpha_{i}\right\rangle=0$, for all $i$. Construct $\left|\Phi^{\prime}\right\rangle$ as follows:

$$
\left|\Phi^{\prime}\right\rangle=|\Phi\rangle+\left|a_{1}\right\rangle\left|b_{1}\right\rangle\left|c_{1}\right\rangle
$$

where $\left|b_{1}\right\rangle \in \mathcal{H}_{B}$ and $\left|c_{1}\right\rangle \in \mathcal{H}_{C}$ are arbitrary non-zero vectors. Note that we can obtain $|\Phi\rangle$ from $\left|\Phi^{\prime}\right\rangle$ by performing the local projection $\left(I-\left|a_{1}\right\rangle\left\langle a_{1}\right|\right)^{A} \otimes I^{B} \otimes I^{C}$. Thus $\operatorname{Sch}\left(\Phi^{\prime}\right) \geq \operatorname{Sch}(\Phi)$, and that $\left|\Phi^{\prime}\right\rangle$ is of the maximal tensor rank $S \operatorname{ch}(\mathcal{H})$. Furthermore we 
have $\operatorname{rank}\left(\rho_{\Phi^{\prime}}^{A}\right)>\operatorname{rank}\left(\rho_{\Phi}^{A}\right)$. If $\left|\Phi^{\prime}\right\rangle$ is of full local rank already, the proof is complete. Otherwise repeat the above arguments. Thus after a finite number of repetitions of the above steps, we can obtain a state of the maximum tensor rank and, of full local ranks, thus maximal.

We construct a maximal state with the tensor rank $d_{1}$ as follows. Take a basis $\left\{\left|a_{k}\right\rangle: k=\right.$ $\left.1, \cdots, d_{1}\right\}$ of $\mathcal{H}_{A}$ and a set of $d_{1}$ linearly independent product vectors $\left\{\left|b_{k}\right\rangle\left|c_{k}\right\rangle: 1 \leq k \leq d_{1}\right\}$ of $\mathcal{H}_{B} \otimes \mathcal{H}_{C}$ and then construct

$$
|\Psi\rangle=\sum_{k=1}^{d_{1}}\left|a_{k}\right\rangle\left|b_{k}\right\rangle\left|c_{k}\right\rangle
$$

Clearly $\operatorname{Sch}(\Psi)=d_{1}$. However, we cannot guarantee that $S c h(\Psi)$ is of full local rank at $B$ and $C$ sides. For instance, $\left\{\left|b_{k}\right\rangle: 1 \leq k \leq d_{1}\right\}$ may not span $\mathcal{H}_{B}$. A simple example is $|0\rangle|00\rangle+|1\rangle|01\rangle$, which is of tensor rank 2 but is not of full rank. One can avoid this problem by using the special construction presented in Ref. [15]. An alternative construction is as follows. Let $\{|0\rangle, \cdots,|d-1\rangle\}$ be a basis for a dimension $d$ space. Consider the state

$$
\sum_{i=0}^{d_{3}-1}|i, i, i\rangle+\sum_{i=d_{3}}^{d_{2}-1}|i, i, 0\rangle+\sum_{i=d_{2}}^{d_{1}-1}\left|i, a_{i}, c_{i}\right\rangle,
$$

where $\left(a_{i}, c_{i}\right)$ 's are distinct elements that do not appear in the first two terms. It is quite straightforward to verify the above state is maximal and has tensor rank $d_{1}$.

We will assume from now on that $d_{1}<d_{2} \cdots d_{n}$, and show that there are at least two incomparable maximal states under this assumption. We will focus on $n=3$ and return to the general case later. Let $k=d_{2} d_{3}-d_{1}$.

First, we prove the result for the case $S \operatorname{ch}(\mathcal{H})>d_{1}$. We then show if $d_{1}<d_{2} d_{3}-1$ then $\operatorname{Sch}(\mathcal{H})>d_{1}$. Finally we show that for $d_{1}=d_{2} d_{3}-1$, there are precisely $\min \left\{d_{2}, d_{3}\right\} \geq 2$ number of maximal equivalence classes.

By Lemma 2.5, if $S c h(\mathcal{H}) \neq d_{1}$ then there are two incomparable maximal states. This is indeed the case when $k>1$.

Lemma 2.6. There are at least two incomparable maximal states in $d_{1} \otimes d_{2} \otimes d_{3}$ if $k>1$.

Proof. By Theorem 1.2, we have $S \operatorname{ch}(\mathcal{H}) \geq d_{1}+1$ for $k \geq 4$, by Item (i), and for $k=2,3$ by Item (ii) (note that when $k=3, \max \left\{d_{2}, d_{3}\right\} \geq k$, since otherwise $d_{1}=d_{2}=d_{3}=k=2$ ). Therefore, when $k>4, S c h(\mathcal{H}) \neq d_{1}$. Since any two equivalent states must have the same tensor rank (by Proposition 2.2), Theorem 1.1 implies that there are two incomparable maximal states.

We now focus on the case $d_{1}=d_{2} d_{3}-1$. By Theorem $1.2($ ii $), \operatorname{Sch}(\Phi)=d_{1}$. Since a maximal state has full local ranks thus having a tensor rank $\geq d_{1}$, its tensor rank must be precisely $d_{1}$. The following lemma completes the proof for our main theorem for $n=3$.

Lemma 2.7. If $d_{1}=d_{2} d_{3}-1$, there are precisely $\min \left\{d_{2}, d_{3}\right\}$ inequivalent maximal states. 
Proof. Let $|\Phi\rangle=\sum_{i=0}^{d_{1}-1}|i\rangle^{A}\left|\phi_{i}\right\rangle^{B C}$ and $|\Psi\rangle=\sum_{i=0}^{d_{1}-1}|i\rangle^{A}\left|\psi_{i}\right\rangle^{B C}$ are two maximal states. Since they are of full local ranks, $\left\{\left|\phi_{i}\right\rangle: i=0 . . d_{1}-1\right\}$ and $\left\{\left|\psi_{i}\right\rangle: i=0 . . d_{1}-1\right\}$ are linear independent. Therefore, there exist two unique (up to a phase factor) non-zero states $\left|\Phi^{\prime}\right\rangle^{B C}$ and $\left|\Psi^{\prime}\right\rangle^{B C}$ such that $\left\langle\Phi^{\prime} \mid \phi_{i}\right\rangle=0$ and $\left\langle\Psi^{\prime} \mid \psi_{i}\right\rangle=0$, for all $i=0 . . d_{1}-1$.

If $|\Phi\rangle$ and $|\Psi\rangle$ are equivalent, there exist invertible operators $L_{1}, L_{2}, L_{3}$ such that $|\Phi\rangle=$ $\left(L_{1} \otimes L_{2} \otimes L_{3}\right)|\Psi\rangle$, implying that

$$
\operatorname{span}\left\{\left|\phi_{i}\right\rangle: 1 \leq i \leq d_{1}\right\}=\left(L_{2} \otimes L_{3}\right) \operatorname{span}\left\{\left|\psi_{i}\right\rangle: 1 \leq i \leq d_{1}\right\}
$$

This is equivalent to

$$
\left|\Phi^{\prime}\right\rangle^{B C}=\left(\left(L_{2}^{\dagger}\right)^{-1} \otimes\left(L_{3}^{\dagger}\right)^{-1}\right)\left|\Psi^{\prime}\right\rangle^{B C}
$$

In other words, $\left|\Phi^{\prime}\right\rangle$ and $\left|\Psi^{\prime}\right\rangle$ are equivalent under SLOCC. Note that $\left|\Phi^{\prime}\right\rangle$ and $\left|\Psi^{\prime}\right\rangle$ are both bipartite pure states. It is well known that two bipartite pure states are SLOCC equivalent if and only if they have the same Schmidt rank. For a non-zero pure state in $d_{2} \otimes d_{3}$, the Schmidt rank may take any values from $1, \ldots, \min \left\{d_{2}, d_{3}\right\}$. Therefore, there are at least $\min \left\{d_{2}, d_{3}\right\}$ different equivalence classes of stochastic maximal states.

On the other hand, if $\left|\Phi^{\prime}\right\rangle$ and $\left|\Psi^{\prime}\right\rangle$ have the same Schmidt rank, there exist invertible $L_{2}$ and $L_{3}$ such that Eqn. (4), and consequently, Eqn. (3) hold. Thus $L_{2} \otimes L_{3}|\Psi\rangle=$ $\sum_{i=0}^{d_{1}-1}\left|\alpha_{i}\right\rangle^{A}\left|\psi_{i}\right\rangle$, for some states $\left|\alpha_{i}\right\rangle^{A}, i=0 . . d_{1}-1$. Those states must be linearly independent, since $L_{2} \otimes L_{3}$ does not change the local rank of $|\Psi\rangle$. Thus setting $L_{1}=\sum_{i=0}^{d_{1}-1}|i\rangle\left\langle\alpha_{i}\right|$, we have that $L_{1}$ is invertible and $|\Phi\rangle=L_{1} \otimes L_{2} \otimes L_{3}|\Psi\rangle$. Thus $|\Phi\rangle$ and $|\Psi\rangle$ are equivalent. Consequently, there are at most $\min \left\{d_{2}, d_{3}\right\}$ number of maximal equivalence class.

An example to illustrate Lemma 2.7 is the state space $\mathcal{H}=\mathcal{H}_{3} \otimes \mathcal{H}_{2} \otimes \mathcal{H}_{2}$. Miyake has obtained all eight equivalence class of this space [16]. Two of these equivalence classes are maximal. The above Lemma provides an alternative method to characterize the maximal states in this space. By the Lemma, there is a one-to-one correspondence between the maximal equivalence class of $\mathcal{H}$ and the equivalence class of $\mathcal{H}^{\prime}=\mathcal{H}_{2} \otimes \mathcal{H}_{2}$. The latter space has precisely two equivalence class with the representatives $\left|\Phi_{1}^{\prime}\right\rangle=|10\rangle$ and $\left|\Phi_{2}^{\prime}\right\rangle=|01\rangle$ $|10\rangle$. As a result, there are only two maximal equivalence class, which can be constructed according to $\left|\Phi_{1}^{\prime}\right\rangle$ and $\left|\Phi_{2}^{\prime}\right\rangle$ as follows:

$$
\begin{aligned}
\left|\Phi_{1}\right\rangle & =|0\rangle|00\rangle+|1\rangle|01\rangle+|2\rangle|11\rangle, \\
\left|\Phi_{2}\right\rangle & =|0\rangle|00\rangle+|1\rangle(|01\rangle+|10\rangle)+|2\rangle|11\rangle .
\end{aligned}
$$

Lemma 2.6 and 2.7 together imply Theorem 1.1.

We have finished the proof of Main Theorem for $n=3$. We deal with the general case below (that is to show that there is no maximum state in $d_{1} \otimes d_{2} \otimes \cdots \otimes d_{n}$ if $d_{1}<d_{2} d_{3} \cdots d_{n}$ ).

Proof of Theorem 1.1. We need only consider $n>3$. Suppose that $n=4$ and $d_{1}<d_{2} d_{3} d_{4}$. Consider the tripartite state space $d_{1} \otimes d_{2} \otimes d_{3} d_{4}$. There are two cases:

Case 1. $d_{3} d_{4}=d_{1} d_{2}$. Since $d_{1} \geq d_{2} \geq d_{3} \geq d_{4}$ we have $d_{1}=d_{2}=d_{3}=d_{4}=d$. One can easily verify that $\left|\Phi_{d}\right\rangle^{A B} \otimes\left|\Phi_{d}\right\rangle^{C D}$ and $\left|\Phi_{d}\right\rangle^{A C} \otimes\left|\Phi_{d}\right\rangle^{B D}$ both are of full local rank $d$, where $\left|\Phi_{d}\right\rangle$ is the generalized EPR state defined in Eqn. 1. Observe that with respect to the $A C: B D$ partition, the former is entangled yet the latter is not, and with respect to the 
$A B$ : $C D$ partition, the opposite holds. Since no LOCC protocol can create entanglement, the two states are not comparable under SLOCC.

Case 2. $d_{3} d_{4}<d_{1} d_{2}$. In this case, applying the result for $n=3$ we know that there are at least two inequivalent maximal states in $d_{1} \otimes d_{2} \otimes d_{3} d_{4}$. By Corollary 2.4, those states are also maximal in $d_{1} \otimes d_{2} \otimes d_{3} \otimes d_{4}$. They remain incomparable under SLOCC with respect to this refined partition.

Suppose that the theorem is correct for $n=k, k \geq 4$. Consider $n=k+1$. Since $k \geq 4, d_{k} d_{k+1}<d_{1} d_{2} d_{3} \cdots d_{k-1}$. By the inductive hypothesis, there are two incomparable maximum states in $d_{1} \otimes d_{2} \otimes \cdots \otimes d_{k-1} \otimes d_{k} d_{k+1}$. By Corollary 2.4, they remain maximal and incomparable in the refinement $\mathcal{H}$. Thus the theorem is correct for $n=k+1$, therefore correct for all $n \geq 4$.

\section{Correspondence between maximal equivalence class and SLOCC equivalences classes}

In this section we consider state spaces such that $d_{1}<d_{2} \cdots d_{K}$. So it is impossible to find one state from which one can locally prepare any other state even probabilistically.

An alternative goal is to characterize all maximal equivalence class. In particular, we ask when a multipartite state space $\mathcal{H}$ has only a finite number of maximal stochastic equivalence classes. Suppose that $\mathcal{H}$ has a finite number of maximal equivalence class with the representative states $\left|\Phi_{1}\right\rangle, \cdots,\left|\Phi_{N}\right\rangle$. Then for any state $|\psi\rangle \in \mathcal{H}$, there exists $1 \leq k \leq N$ such that $\left|\Phi_{k}\right\rangle$ can be converted into $|\psi\rangle$ by SLOCC. So the set of states $\left\{\left|\Phi_{1}\right\rangle, \cdots,\left|\Phi_{N}\right\rangle\right\}$ is able to locally prepare any other state in $\mathcal{H}$ with nonzero probability. In practice, we only need to prepare the set of maximal states $\left\{\left|\Phi_{k}\right\rangle\right\}$ and then create other states using SLOCC. Thus identifying the maximal equivalence classes for a given space is highly desirable.

For the sake of convenience, from now on we mainly focus on tripartite state space. Most of our results are also valid for the case of $K>3$. We assume that $d_{1}=d_{2} d_{3}-k$, where $k<d_{2} d_{3} / 2$. We shall employ a correspondence between the maximal equivalence class of $\mathcal{H}_{d_{1}} \otimes \mathcal{H}_{d_{2}} \otimes \mathcal{H}_{d_{3}}$ and the equivalence class of $\mathcal{H}_{k} \otimes \mathcal{H}_{d_{2}} \otimes \mathcal{H}_{d_{3}}$.

Definition 3.1. Let $|\Phi\rangle \in \mathcal{H}_{d_{1}} \otimes \mathcal{H}_{d_{2}} \otimes \mathcal{H}_{d_{3}}$ and $\operatorname{rank}\left(\rho_{\Phi}^{A_{1}}\right)=d_{1}$, write $|\Phi\rangle=\sum_{i=1}^{d_{1}}|i\rangle^{A_{1}}\left|\phi_{i}\right\rangle^{A_{2} A_{3}}$, where $\left\{|i\rangle^{A_{1}}: 1 \leq i \leq d_{1}\right\}$ is any basis for $\mathcal{H}_{d_{1}}$. Let $\mathcal{T}^{A_{1}}(\Phi)$ be the SLOCC equivalence class of $\mathcal{H}_{k} \otimes \mathcal{H}_{d_{2}} \otimes \mathcal{H}_{d_{3}}$ with representative state $\left|\Phi^{\prime}\right\rangle=\sum_{i=1}^{k}|i\rangle^{A_{1}}\left|\phi_{i}^{\perp}\right\rangle^{A_{2} A_{3}}$, where $\left\{|i\rangle^{A_{1}}: 1 \leq\right.$ $i \leq k\}$ is a basis for $\mathcal{H}_{k}$ and $\left\{\left|\phi_{i}^{\perp}\right\rangle: 1 \leq i \leq k\right\}$ is any basis for $\operatorname{span}^{\perp}\left\{\left|\phi_{i}\right\rangle^{A_{2} A_{3}}: 1 \leq i \leq d_{1}\right\}$.

It is easy to see that $\mathcal{T}^{A_{1}}(\Phi)$ is well-defined in the sense that it does not depend on which basis of $\operatorname{span}^{\perp}\left\{\left|\phi_{i}\right\rangle^{A_{2} A_{3}}: 1 \leq i \leq d_{1}\right\}$ we choose. It is also worth noting that any state in $\mathcal{T}^{A_{1}}(\Phi)$ should be of local rank $k$ between $A_{1}$ and $A_{2} A_{3}$.

The importance of the map $\mathcal{T}^{A_{1}}$ is due to the following lemma, which can be treated as a generalization of Lemma 2.7.

Lemma 3.2. Let $|\Phi\rangle$ and $|\Psi\rangle$ be two vectors in $\mathcal{H} \operatorname{such}$ that $\operatorname{rank}\left(\rho_{\Phi}^{A_{1}}\right)=\operatorname{rank}\left(\rho_{\Psi}^{A_{1}}\right)=d_{1}$. Then $|\Phi\rangle$ and $|\Psi\rangle$ are equivalent under SLOCC if and only if $\mathcal{T}^{A_{1}}(\Phi)=\mathcal{T}^{A_{1}}(\Psi)$.

Proof. The proof idea is similar to Lemma 2.7. For completeness, we present a detailed proof here. By Lemma 2.1, $|\Phi\rangle$ and $|\Psi\rangle$ are equivalent under SLOCC if and only if there 
are invertible linear operators $L_{1}, L_{2}, L_{3}$ such that $|\Phi\rangle=\left(L_{1} \otimes L_{2} \otimes L_{3}\right)|\Psi\rangle$. More explicitly, we have

$$
\sum_{i=1}^{d_{1}}|i\rangle^{A_{1}}\left|\phi_{i}\right\rangle^{A_{2} A_{3}}=\left(L_{1} \otimes L_{2} \otimes L_{3}\right) \sum_{i=1}^{d_{1}}|i\rangle^{A_{1}}\left|\psi_{i}\right\rangle^{A_{2} A_{3}} .
$$

Applying $\left\langle\left. j\right|^{A_{1}} \otimes I^{A_{2} A_{3}}\right.$ to both sides of the above equation, we have

$$
\left|\phi_{j}\right\rangle^{A_{2} A_{3}}=\left(L_{2} \otimes L_{3}\right) \sum_{i=1}^{d_{1}}\left\langle j\left|L_{1}\right| i\right\rangle\left|\psi_{i}\right\rangle^{A_{2} A_{3}} .
$$

That means

$$
\left|\phi_{j}\right\rangle^{A_{2} A_{3}} \in\left(L_{2} \otimes L_{3}\right) \operatorname{span}\left\{\left|\psi_{i}\right\rangle^{A_{2} A_{3}}: 1 \leq i \leq d_{1}\right\}
$$

for each $1 \leq j \leq d_{1}$. Noticing further that $L_{1}$ is invertible, we have

$$
\operatorname{span}\left\{\left|\phi_{i}\right\rangle^{A_{2} A_{3}}\right\}=\left(L_{2} \otimes L_{3}\right) \operatorname{span}\left\{\left|\psi_{i}\right\rangle^{A_{2} A_{3}}\right\} .
$$

Conversely, we can readily show that the existence of invertible linear operators $L_{2}$ and $L_{3}$ such that Eqn. (77) holds also implies the SLOCC equivalence between $|\Phi\rangle$ and $|\Psi\rangle$. It is easy to verify Eqn. (17) can be rewritten into the following

$$
\operatorname{span}^{\perp}\left\{\left|\phi_{i}\right\rangle^{A_{2} A_{3}}\right\}=\left(\left(L_{2}^{\dagger}\right)^{-1} \otimes\left(L_{3}^{\dagger}\right)^{-1}\right) \operatorname{span}^{\perp}\left\{\left|\psi_{i}\right\rangle^{A_{2} A_{3}}\right\} .
$$

Using a similar argument, we can show the above equation means that $\left|\Phi^{\prime}\right\rangle=\sum_{i=1}^{k}|i\rangle^{A_{1}}\left|\phi_{i}^{\perp}\right\rangle^{A_{2} A_{3}}$ and $\left|\Psi^{\prime}\right\rangle=\sum_{i=1}^{k}|i\rangle^{A_{1}}\left|\psi_{i}^{\perp}\right\rangle^{A_{2} A_{3}}$ are equivalent. In other words, $\mathcal{T}^{A_{1}}(\Phi)$ and $\mathcal{T}^{A_{1}}(\Psi)$ coincide.

When $k<d_{2} d_{3} / 2$, we have $k<d_{1}$. It may be much easier to decide the SLOCC equivalence between $\mathcal{T}^{A_{1}}(\Phi)$ and $\mathcal{T}^{A_{1}}(\Psi)$ than that between $|\Phi\rangle$ and $|\Psi\rangle$. However, $\mathcal{T}^{A_{1}}$ is not a one-to-one correspondence between the maximal equivalence class of $\mathcal{H}_{d_{1}} \otimes \mathcal{H}_{d_{2}} \otimes \mathcal{H}_{d_{3}}$ and the equivalence class of $\mathcal{H}_{k} \otimes \mathcal{H}_{d_{2}} \otimes \mathcal{H}_{d_{3}}$. In general, the image of $\mathcal{T}^{A_{1}}$ is only a proper subset of $\mathcal{H}_{k} \otimes \mathcal{H}_{d_{2}} \otimes \mathcal{H}_{d_{3}}$. Fortunately, in the special case of $k=1$, we do have a one-to-one correspondence as stated below. The case of $n=3$ was proved in in Lemma 2.7.

Theorem 3.3. There is a one-to-one correspondence between the maximal equivalence class in $\mathcal{H}_{d_{1}} \otimes \cdots \otimes \mathcal{H}_{d_{K}}$ and the stochastic equivalence classes of $\mathcal{H}_{d_{2}} \otimes \cdots \otimes \mathcal{H}_{d_{K}}$, where $d_{1}=d_{2} \cdots d_{K}-1$.

The following theorem also follows directly from Lemma 3.2 .

Theorem 3.4. If $\mathcal{H}_{k} \otimes \mathcal{H}_{d_{2}} \otimes \cdots \otimes \mathcal{H}_{d_{K}}$ has a finite number of equivalence class, then $\mathcal{H}_{d_{1}} \otimes \mathcal{H}_{d_{2}} \otimes \cdots \otimes \mathcal{H}_{d_{K}}$ has a finite number of maximal equivalence class.

Using the known result that there are a finite number of equivalence class for tripartite systems of the dimensions $d_{3}=2, d_{2} \leq 3$ [14], we have the following corollary.

Corollary 3.5. Each of the following spaces has a finite number of maximal equivalence class: $(2 n-2) \otimes n \otimes 2,(2 n-3) \otimes n \otimes 2,(3 n-2) \otimes n \otimes 2$, and, when $2 \leq \min \{m, n\} \leq 3$, $(2 m n-1) \otimes m \otimes n \otimes 2$. 
For $\mathcal{H}=\mathcal{H}_{7} \otimes \mathcal{H}_{2} \otimes \mathcal{H}_{2} \otimes \mathcal{H}_{2}$, it follows from the above Corollary that it has a finite number of maximal equivalence class. In contrast, $\mathcal{H}$ has an infinite number of equivalence class [18]. Another notable case is $\mathcal{H}=\mathcal{H}_{4} \otimes \mathcal{H}_{3} \otimes \mathcal{H}_{2}$. We know from [16] that $\mathcal{H}^{\prime}=\mathcal{H}_{2} \otimes \mathcal{H}_{3} \otimes \mathcal{H}_{2}$ has 8 equivalence class. Thus by Theorem $3.4, \mathcal{H}$ has at most 8 different maximal equivalence classes. However, the exact number is strictly smaller than 8 as some equivalence classes do not correspond to any equivalence classes. A careful investigation shows that $\mathcal{H}_{4} \otimes \mathcal{H}_{3} \otimes \mathcal{H}_{2}$ has exactly 5 maximal equivalence classes.

\section{Discussions and open problems}

We showed as our main result that a multipartite quantum system is allowed to have a maximum entangled state only when there is a subsystem whose dimension is no less than the total dimension of the rest of the system. When this condition does not hold, there are multiple distinct maximal equivalence classes. A complete classification of those maximal states would be of great value, both theoretically and practically. To this end, we provided a connection between the maximal equivalence classes in a state space with the stochastic equivalence classes in another state space of a smaller dimension. In particular, we proved that when $d_{1}=d_{2} \cdots d_{K}-1$, there is a one-to-one correspondence between the maximal equivalence classes of $\mathcal{H}_{d_{1}} \otimes \cdots \otimes \mathcal{H}_{d_{K}}$ and the stochastic equivalence classes of $\mathcal{H}_{d_{2}} \otimes \cdots \otimes \mathcal{H}_{d_{K}}$. Various examples are studied to demonstrate the applications of these results.

We conclude by proposing two directions for further investigations that we consider of both theoretical and practical importance. The first is to understand deeper the structure of of partial orders on LOCC and equivalence class. Structural results will not only deepen our understanding of entanglement, but will also find applications for establishing multipartite entanglement when there is no maximum state.

For example, which spaces have an infinite number of SLOCC equivalence classes, or an infinite number of maximal classes? For those spaces having a finite number of maximal equivalence classes, the parties can share some number of each maximal states, and use them later to generate arbitrary desired states. Note that in this case the ratio of the output states and the initial states will not be as efficient as the case when a maximum state exists, unless the distribution of the output states is known in advance. A second and related question is, given a space that does not admit a maximum state, what is the "smallest" state outside the specified space yet is able to generate an arbitrary state in that space? For instance, there are two maximal equivalence classes in $2 \otimes 2 \otimes 3$, represented by the states $\left|\Phi_{1}\right\rangle$ and $\left|\Phi_{2}\right\rangle$ in Eqn. (5). Either state, however, can generate any state from $2 \otimes 2 \otimes 2$ through SLOCC.

A second direction is to consider approximate generation of entangled states. Are there spaces that do not have a maximum state but have an "approximate" maximum state in the sense that all other states can be approximated to an arbitrary small precision through a LOCC protocol on that state? Such an approximate state is as good as the precise state in practice. Consider another setting where the parties wish to generate a large number of a target state. A solution is for them to share in bulk some initial state, since many copies of a fixed state are likely to be cheaper to manufacture. A natural question is, which initial state will offer the most efficient rate of conversion in the worst case (over all possible 
target states)? In particular, which spaces admit the best possible ratio of 1 asymptotically? Perhaps the notion of "border rank" (C.f. Chapter 15 in [8]), the approximate version of tensor rank, in algebraic complexity theory may be useful for tackling those intriguing problems.

\section{Acknowledgments}

We are grateful to Eric Chitambar and Zhengfeng Ji for helpful discussions and comments.

\section{References}

[1] A. Einstein, B. Podolsky, and N. Rosen, Phys. Rev. 47, 777 (1935).

[2] R. Horodecki, P. Horodecki, M. Horodecki, and K. Horodecki, Rev. Mod. Phys. 81, 865 (2009).

[3] O. Gühne and G. Tóth, Physics Reports 474, 1 (2009).

[4] C. H. Bennett, G. Brassard, C. Crépeau, R. Jozsa, A. Peres, and W. K. Wootters, Phys. Rev. Lett. 70, 1895 (1993).

[5] G. Vidal, J. Mod. Opt. 47, 355 (2000).

[6] W. Dür, G. Vidal, and J. I. Cirac, Phys. Rev. A 62, 062314 (2000).

[7] C. H. Bennett, S. Popescu, D. Rohrlich, J. A. Smolin, and A. V. Thapliyal, Phys. Rev. A 63, 012307 (2000).

[8] P. Bürgisser, M. Clausen, and M. A. Shokrollahi, Algebraic complexity theory, Springer, 1997.

[9] J. Eisert, H.-J. Briegel,Phys. Rev. A 64, 022306 (2001).

[10] E. Chitambar, R. Duan, and Y. Shi, Phys. Rev. Lett. 101, 140502 (2008).

[11] J. von zur Gathen, Linear algebra and its applications 144, 49 (1991).

[12] N. H. Bshouty, SIAM J. Comp. 19, 467 (1990).

[13] H.-K. Lo and S. Popescu, Phys. Rev. A 63, 022301 (2001), see also quant-ph/9707038.

[14] L. Chen, Y.-X. Chen, and Y.-X. Mei, Phys. Rev. A 74, 052331 (2006).

[15] R. Duan, Y. Feng, Z. Ji, and M. Ying, Phys. Rev. Lett. 98, 230502 (2007).

[16] A. Miyake, Phys. Rev. A 67, 012108 (2003).

[17] S. Popescu, Phys. Rev. Lett. 74, 2619 (1995).

[18] F. Verstraete, J. Dehaene, B. De Moor, and H. Verschelde, Phys. Rev. A 65, 052112 (2002). 